\title{
Nuevos registros de Balistes polylepis (Balistidae), Sphoeroides lobatus (Tetraodontidae), Mola mola y M. ramsayi (Molidae) en San Antonio, Chile (Pisces, Tetraodontiformes)
}

\author{
José L. Brito \\ Museo Municipal de Ciencias Naturales y Arqueología de San Antonio \\ Sanfuentes 2365, Barrancas, San Antonio, Chile \\ E-mail:museo_imsa@hotmail.com
}

\begin{abstract}
RESUMEN. El hallazgo de tres ejemplares de Balistes polylepis Steindachner, 1876; un ejemplar de Sphoeroides lobatus Steindachner, 1870 y un ejemplar de Mola mola Linnaeus, 1758, en la costa de San Antonio y tres ejemplares de $M$. ramsayi (Giglioli, 1833), permite aumentar la distribución geográfica conocida de estas especies desde el norte hasta la costa central de Chile. La presencia de estas especies está asociada con los efectos de los fenómenos El Niño 1982-83 y 1997-98.

Palabras claves: Balistes polylepis, Sphoeroides lobatus, Mola mola, Mola ramsayi, Tetraodontiformes, El Niño, Chile central.

\section{New records of Balistes polylepis (Balistidae), Sphoeroides lobatus (Tetraodontidae), Mola mola and M. ramsayi (Molidae) from San Antonio, Chile (Pisces, Tetraodontiformes)}

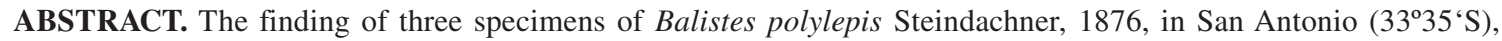

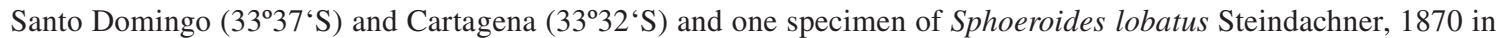
Matanza (33'58'S) and one specimen of Mola mola Linnaeus, 1758 and three specimens of M. ramsayi (Giglioli, $1833)$, to the west of Las Cruces ( $33^{\circ} 31^{\prime} \mathrm{S}$ ) increases the known geographic distribution for these species from northern Chile to central Chile. Their presence is associated to El Niño phenomena of 1982-1983 and 1997-1998.
\end{abstract}

Key words: Balistes polylepis, Sphoeroides lobatus, Mola mola, Mola ramsayi, Tetraodontiformes, El Niño, central Chile.

Los peces Tetraodontiformes se distinguen de otros teleósteos principalmente por sus fuertes mandíbulas y una boca muy pequeña, provista de poderosos incisivos o pico afilado compuesto por varios dientes modificados (Bond, 1979, 1997). Aunque en los océanos las especies de este orden son numerosas, en Chile son escasas, probablemente debido a que su hábitat se encuentra en los mares cálidos y ligados a arrecifes de coral (Moyle \& Cech, 1982; Springer, 1982). En Chile, los Tetraodontiformes están representados por 22 especies distribuidas en seis familias: Balistidae, Ostraciidae, Tetraodontidae, Diodontidae, Monacanthidae y
Molidae (Bahamonde \& Pequeño, 1975; Pequeño, 1989, 1997).

El presente trabajo tiene por objetivo documentar nuevos registros de Balistes polylepis, Sphoeroides lobatus, Mola mola y M. ramsayi, y extender la distribución geográfica conocida para las tres primeras especies indicadas, sobre la base de ejemplares capturados en la zona central de Chile. Los especímenes analizados se encuentran depositados en la colección ictiológica del Museo Municipal de Ciencias Naturales y Arqueología de San Antonio (MMSA). 
Balistes polylepis Steindachner, 1876

Es una de las siete especies de Balistidae presente en aguas chilenas, siendo endémica del Pacífico oriental. Su distribución se extiende desde San Francisco, California (USA), hasta islas Galápagos (Ecuador) y Lobos de Afuera (Perú) (Hildebrand, 1946; Berry \& Baldwin, 1966; Chirichigno, 1974; Vélez et al., 1984; Chirichigno \& Vélez, 1998). También se ha registrado frente a Iquique $\left(20^{\circ} 16^{\prime} \mathrm{S}\right)$ en 1957 y 1984 (De Buen, 1959; Kong et al., 1985) y a Antofagasta (2339'S) en 1977, 1983 y 1984 (Meléndez et al., 1993; Kong et al., 1985; Kong \& Bolados, 1987).

Material estudiado: MMSA-PE-129, un ejemplar, $375 \mathrm{~mm}$ de longitud total (LT), capturado en marzo de 1988 frente a San Antonio (Fig. 1). MMSA-PE-433, un especimen de $455 \mathrm{~mm}$ de LT, capturado el 7 abril de 1998 a 0,5 mn al oeste de San Antonio. MMSA-PE-473, un ejemplar de 366 mm de LT, capturado el 11 de enero de 1999 al oeste de Cartagena $\left(33^{\circ} 32^{\prime} \mathrm{S}\right)$. Los caracteres morfométricos y merísticos de estos ejemplares se entregan en la Tabla 1.

Descripción: cuerpo alto y pedúnculo caudal comprimido. Cabeza grande con el perfil casi recto; hocico corto y terminal, abertura bucal del tamaño del diámetro ocular. Labios bien marcados, detrás de ellos se aprecia un pliegue transversal, seguido de un espacio semilunar, donde la piel está completamente escamada y con algunas espinas. Posee cuatro escamas grandes en la zona posterior al margen de la cubierta branquial. Primera aleta dorsal con la primera espina gruesa, alta, y con granulaciones en la parte anterior y los costados. Le siguen otras dos

Tabla 1. Caracteres morfométricos ( $\mathrm{mm})$ y merísticos de los tres ejemplares de Balistes polylepis comparados con el ejemplar descrito por De Buen (1959). Las mediciones se hicieron siguiendo los criterios de Fischer \& Hureau (1985) y Matsuura (1980), para Balistidae.

Table 1. Morphometric ( $\mathrm{mm})$ and meristic characters of three specimens of Balistes polylepis compared with the specimen described by De Buen (1959). The measurements were made according to Fischer \& Hureau (1985) and Matsuura (1980), for Balistidae.

\begin{tabular}{|c|c|c|c|c|}
\hline Número de catálogo & MMSA-PE-129 & MMSA-PE-433 & MMSA-PE-473 & De Buen (1959) \\
\hline Longitud total & 375 & 455 & 366 & 420 \\
\hline Longitud estándar & 298 & 342 & 305 & 325 \\
\hline Longitud cabeza & 98 & 100 & 93 & \\
\hline Distancia preorbital & 60 & 71 & 62 & 82 \\
\hline Diámetro orbital & 19 & 18 & 27 & 19 \\
\hline Distancia postorbital & 13 & 16 & 18 & 29 \\
\hline Distancia interorbital & 29 & 34 & 32 & 34 \\
\hline Longitud predorsal & 72 & 89 & 85 & \\
\hline Distancia prepectoral & 90 & 102 & 101 & \\
\hline Distancia preanal & 171 & 187 & 165 & \\
\hline Distancia prepélvica & 121 & 132 & 140 & \\
\hline Base dorsal & 65 & 78 & 78 & \\
\hline Base aleta dorsal con espinas & 49 & 59 & 52 & \\
\hline Base anal & 93 & 107 & 94 & \\
\hline Base pectoral & 21 & 24 & 23 & \\
\hline Base pélvica & 34 & 47 & 27 & \\
\hline Longitud pectoral & 43 & 48 & 44 & \\
\hline Longitud pélvica & 34 & 47 & 27 & \\
\hline Altura pedúnculo caudal & 24 & 26 & 25 & \\
\hline $\mathrm{N}^{\mathrm{o}}$ espinas $1^{\mathrm{era}}$ dorsal & III & III & III & III \\
\hline $\mathrm{N}^{\circ}$ radios aleta dorsal & 28 & 28 & 27 & 27 \\
\hline $\mathrm{N}^{\circ}$ radios aleta anal & 26 & 26 & 25 & 25 \\
\hline $\mathrm{N}^{\circ}$ radios aleta pectoral & 14 & 14 & 14 & 15 \\
\hline $\mathrm{N}^{\circ}$ radios aleta pélvica & $1-8$ & $1-12$ & $1-12$ & \\
\hline
\end{tabular}




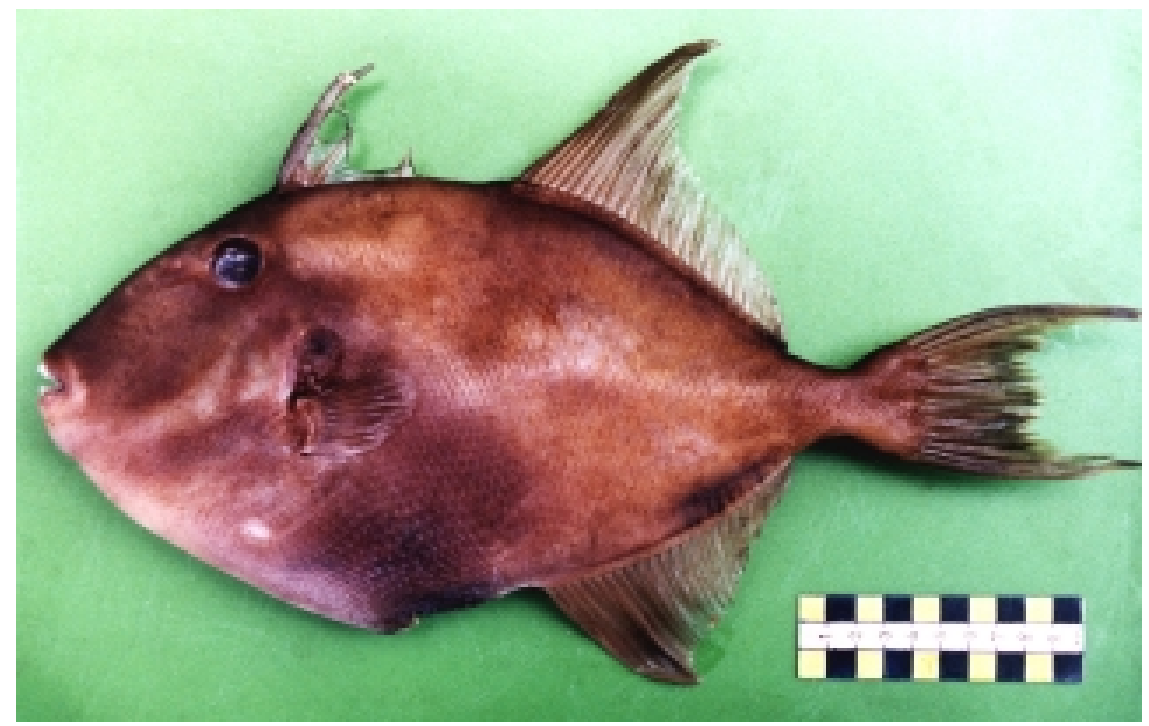

Figura 1. Balistes polylepis Steindachner, 1876, capturado en San Antonio en marzo de 1988. 375 mm de LT (MMSA-PE-129).

Figure 1. Balistes polylepis Steindachner, 1876, captured in San Antonio in March 1988. 375 mm of LT (MMSAPE-129).

espinas fuertes, pero más pequeñas. Aleta dorsal posterior con los radios anteriores más altos y todos ramificados; aletas pectorales cortas con la base ancha y el margen redondeado; aleta ventral con una espina corta y gruesa, con granulaciones, aleta caudal bicóncava y con los extremos de los lóbulos superior e inferior alargados. El color en fresco de los tres ejemplares era gris amarillento-verdoso y más claro en la zona ventral. En las partes distales de la segunda dorsal y anal de color rojo; en formol era gris uniforme y más claro en la zona ventral.

Comentarios: los tres ejemplares analizados tenían características similares al individuo de Iquique, descrito por De Buen (1959) como su subespecie $B$. polylepis rubicundus y coincidieron también con las diagnosis de Chirichigno (1974) y Chirichigno \& Vélez (1998). La principal característica para diferenciarlo de Pseudobalistes naufragium (Jordan \& Starks, 1895), dado a conocer en Chile por Kong \& Bolados (1987), es su hocico completamente escamado, pues $P$. naufragium posee un área desnuda desprovista de escamas sobre el hocico, detrás de la mandíbula. Balistes polylepis, fue descrito para Chile, como una subespecie (De Buen, 1959) denominada B. polylepis rubicundus, pero autores posteriores al examinar y estudiar a otros ejemplares, al parecer no encontraron las características descri- tas por De Buen (1959) y prefirieron dejarlos como B. polylepis (Kong et al., 1985; Meléndez et al., 1993; Kong \& Bolados, 1987), criterio que se mantiene en este trabajo. El hallazgo de estos ejemplares, permite aumentar su distribución geográfica desde Iquique hasta Cartagena y San Antonio $\left(33^{\circ} 35^{\circ} \mathrm{S}\right)$, extendiendo su rango de distribución en casi diez grados de latitud hacia el sur $(1.962 \mathrm{~km})$. Las fechas de los hallazgos permiten relacionar sus registros con los efectos de El Niño 1987 y 1997-98 y el registro de un ejemplar (MMSA-PE-129) en marzo de 1988 y otro (MMSA-PE-473) en enero de 1999 , podría deberse a que los ejemplares tal vez permanecieron atrapados en bolsones de aguas más temperadas o bien resistieron las temperaturas más frías de la costa de San Antonio. Las medidas de estos ejemplares se entregan en la Tabla 1 y se comparan con las del ejemplar descrito por De Buen (1959).

\section{Sphoeroides lobatus Steindachner, 1870}

Es uno de los siete representantes de la familia Tetraodontidae (Pequeño, 1989), y se ha registrado desde el Golfo de California, México, hasta Pucusana, Perú (Vélez et al., 1984), e islas Galápagos (Chirichigno, 1974), y en Chile frente a

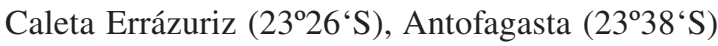


y Taltal (253' $\mathrm{S})$, entre agosto de 1983 y enero de 1984 (Kong et al., 1985; Kong \& Bolados, 1987).

Material estudiado: MMSA-PE-145, un ejemplar de $230 \mathrm{~mm}$ de LT aproximadamente (solo se conservó un dibujo del ejemplar y la piel de la zona abdominal), encontrado varado en el verano de 1983 en playa Salinas, Matanzas (335' $\mathrm{S}$ ).

Descripción: cuerpo alargado y ampliamente redondeado en sección transversal. Con abundantes espinas en el vientre, más pequeñas en el resto del cuerpo. Hocico corto y boca pequeña y terminal. Vientre de color blanco. Cabeza bastante grande en comparación con el cuerpo; con una sola aleta dorsal. Con dos pequeñas formaciones dermales en el dorso más cercanas a los ojos que a la aleta dorsal. El color de la piel conservada disecada es amarillento claro.

Comentarios: la presencia de las formaciones dérmicas dorsal y la piel ventral abundantemente cubierta de espinas, permiten señalar a este ejemplar como Sphoeroides lobatus, coincidiendo con las características entregadas para la especie por Chirichigno (1974), Chirichigno \& Vélez (1998), Meek \& Hildebrand (1928) y Kong \& Bolados (1987). El hallazgo incrementa la extensión de su distribución geográfica en casi ocho grados de latitud hacia el sur, desde Taltal $\left(25^{\circ} 38^{`} \mathrm{~S}\right)$ hasta Matanzas en Chile central, siendo posible asociar su presencia a los efectos del fenómeno "El Niño" 1982-83. El hallazgo del ejemplar varado muerto en una playa, podría indicar que este ejemplar no se haya adaptado a las temperaturas mas frías de las aguas la costa central.

Mola mola Linnaeus, 1758 y $M$. ramsayi (Giglioli, 1833), son dos de los cinco representantes de la familia Molidae en Chile (Pequeño, 1989, 1997). La primera de las especies nombrada fue registrada en el norte de Chile (Kong et al., 1987) y la segunda fue considerada la única especie de este género presente en el país (De Buen, 1957; Bahamonde \& Pequeño, 1975).

\section{Mola mola Linnaeus, 1758}

Material estudiado: MMSA-PE-361 un ejemplar disecado de $955 \mathrm{~mm}$ de LT, capturado con espinel para la pesca de merluza al oeste de Las Cruces (33²9`S), 20 enero 1997.

Descripción: cuerpo discoidal, carente de aletas ventrales, destacando sus enormes aletas dorsal y anal, únicas y de forma triangular sin espinas, aleta caudal gephyrocerca, aleta caudal con doce radios, de los cuales nueve terminan en las placas óseas marginales. Carece de pedúnculo caudal. Boca pequeña con dos dientes mandibulares soldados, recubierto por una piel consistente y provista de pequeños dentículos dérmicos.

Comentarios: las nueve terminaciones o plaquitas óseas dérmicas de la aleta caudal, dispuestas espaciadamente y separadas entre ellas por notables intervalos, pero nunca más amplios que su propia longitud. Las aletas dorsal y anal son más cortas que en los ejemplares estudiados de $M$. ramsayi y también las bases de estas aletas (más anchas en $M$. mola), permiten identificar al ejemplar como $M$. mola. Este registro constituye el segundo para la costa chilena y hasta ahora el más austral conocido, pues permite aumentar su distribución geográfica desde Antofagasta (Kong et al., 1985) en casi diez grados, hasta Las Cruces (3331 'S) en Chile central. Su presencia podría estar asociada también a los efectos de El Niño 199798. Las medidas y recuentos de las estructuras del ejemplar se entregan en la Tabla 2.

\section{Mola ramsayi (Giglioli, 1833)}

Material estudiado: un ejemplar (no conservado) de $870 \mathrm{~mm}$ de LT, capturado al oeste de San Antonio (33³5 'S), 30 marzo 1984; un ejemplar disecado de 650 mm de LT, MMSA-PE-139, capturado en Santo Domingo (333' S), 11 noviembre 1997; un ejemplar disecado de 944 mm de LT, MMSA-PE-301, capturado al oeste de San Antonio y donado al Museo de San Antonio MMSA por la Pesquera San Bernardo, 26 agosto 1995; un ejemplar de $600 \mathrm{~mm}$ de LT, capturado al oeste de Las Cruces, 20 enero 1997 (no conservado); un ejemplar disecado de 900 mm de LT, MMSA-PE-385, varado muerto en playa de Santo Domingo y donado por pescadores deportivos, 6 agosto 1997.

Descripción: color general gris plateado o café oscuro, con manchas más oscuras, vientre blanco con manchas oscuras, la piel estaba cubierta por una capa de mucosidad. Los tres ejemplares tenían 17 radios en la aleta caudal, pero solo los de mayores tallas coinciden en tener 12 de los radios terminados en placas marginales; mientras que el ejemplar juvenil (MMSA-PE-139), sólo tenía cuatro terminaciones en placas. Esta diferencia probablemente se deba a cambios producidos en el desarrollo. La piel disecada es de color café oscuro casi negro en el dorso y gris en los costados (pierde las manchas). 
Tabla 2. Medidas de los ejemplares (mm) de Mola mola y M. ramsayi estudiados.

Table 2. Measurements of the studied specimens (mm) of Mola mola and M. ramsayi.

\begin{tabular}{|c|c|c|c|c|}
\hline Especie & Mola mola & & Mola ramsayi & \\
\hline $\mathrm{N}^{0}$ ejemplar & MMSA-PE-361 & MMSA-PE-139 & MMSA-PE-301 & MMSA-PE-385 \\
\hline Longitud total & 955 & 650 & 944 & 900 \\
\hline Longitud estándar & 775 & 600 & 868 & 790 \\
\hline Longitud cabeza & 305 & 215 & 335 & 262 \\
\hline Distancia preorbital & 135 & 90 & 128 & 115 \\
\hline Diámetro orbital & 42 & 45 & 61 & 48 \\
\hline Distancia postorbital & 96 & 92 & 85 & 97 \\
\hline Distancia interorbital & 175 & 118 & 140 & 127 \\
\hline Longitud predorsal & 545 & 423 & 623 & 510 \\
\hline Longitud prepectoral & 323 & 245 & 338 & 305 \\
\hline Longitud preanal & 510 & 430 & 590 & 625 \\
\hline Base dorsal & 200 & 120 & 189 & 170 \\
\hline Base anal & 198 & 170 & 145 & 160 \\
\hline Base pectoral & 74 & 41 & 91 & 115 \\
\hline Longitud pectoral & 133 & 90 & 145 & 116 \\
\hline Altura aleta dorsal & 390 & 310 & 505 & 426 \\
\hline Altura aleta anal & 420 & 300 & 500 & 420 \\
\hline $\mathrm{N}^{\circ}$ radios aleta pectoral & 12 & 12 & 12 & 12 \\
\hline $\mathrm{N}^{\mathrm{o}}$ radios aleta dorsal & 16 & 17 & 17 & 17 \\
\hline $\mathrm{N}^{\circ}$ radios aleta anal & 16 & 17 & 17 & 17 \\
\hline $\mathrm{N}^{\mathrm{o}}$ radios aleta caudal & 12 & 16 & 16 & 16 \\
\hline $\begin{array}{l}\mathrm{N}^{\circ} \text { terminaciones } \\
\text { dermales aleta caudal }\end{array}$ & 9 & 4 & 12 & 12 \\
\hline
\end{tabular}

Comentarios: los ejemplares de esta especie previamente registrados en Chile fueron estudiados por Philippi (1892), Reed (1897), Delfín (1899), Porter (1904), Gotschlich (1913), Quijada (1913), Oliver (1930, 1943), Fowler (1945, 1951), Mann (1954), De Buen (1957), Bahamonde (1963), Atria (1967), Pequeño (1984), Villalba \& Fernández (1985) para aguas entre Chañaral y Chiloé, y también para isla de Pascua (De Buen, 1959; Villalba \& Fernández, 1985). De Buen (1957), revisó los ejemplares capturados en la costa chilena que se encontraban en algunas colecciones en el país, determinando que la especie es $M$. ramsayi, que posee 16 radios en la aleta caudal, 12 de los cuales se apoyan en las placas marginales y los radios caudales son más largos que la separación entre ellos.

Las aletas dorsal y anal de M. ramsayi son más largas y sus bases son notablemente más angostas que las de M. mola. Estos registros se encuentran dentro de los rangos de distribución geográfica conocidos para esta especie en aguas chilenas. Un ejemplar de $M$. ramsayi de $600 \mathrm{~mm}$ de LT analizado en enero de 1997, presentó varios ectoparásitos identificados como Capsala martinierei Bosc, 1811 (Monogenea, Capasalidae), especie que ya había sido descrita anteriormente en M. ramsayi (Villalba \& Fernández, 1985). Por otra parte, el ejemplar MMSAPE-301, presentó en su sistema digestivo, desde la boca hasta el estómago unos 4.000 céstodos no identificados de entre $0,5 \mathrm{~mm}$ y $5 \mathrm{~cm}$ de longitud. El ejemplar analizado en marzo de 1984, tenía una gran protuberancia globosa bajo la boca, lo cual no ha sido observado en otros ejemplares, lamentablemente este espécimen no se conservó y solo se pudo obtener un dibujo del ejemplar (Fig. 2). Las medidas y recuentos de los ejemplares se entregan en la Tabla 2. 


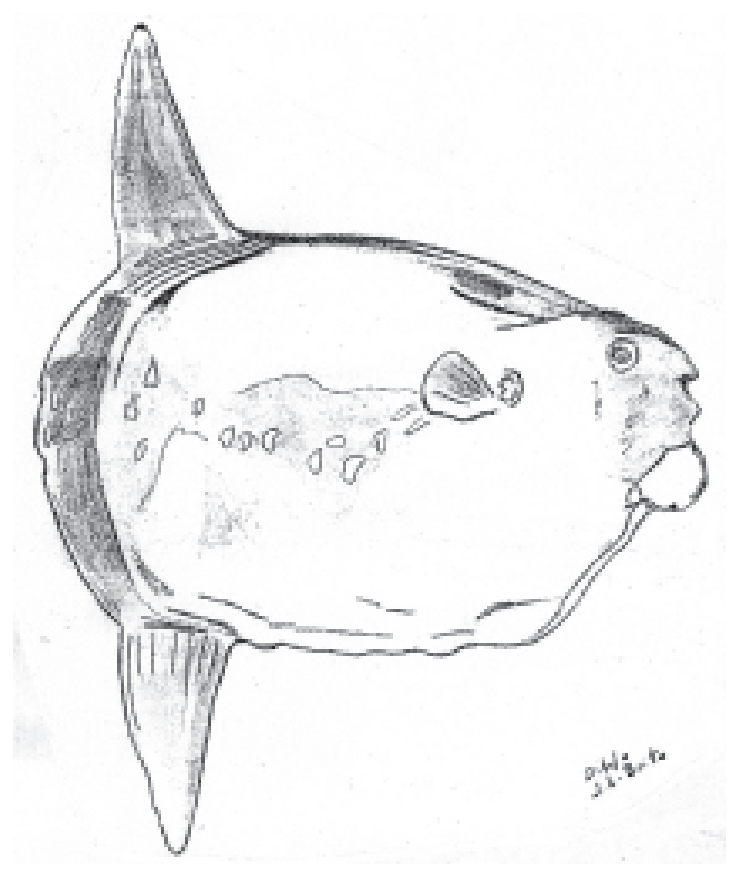

Figura 2. Esquema básico de Mola ramsayi (Giglioli, 1833), capturado al oeste de San Antonio, 870 mm de LT, estudiado en marzo de 1984 (no conservado). Bajo su boca se observa una protuberancia de tipo globosa.

Figure 2. Basic outline of Mola ramsayi (Giglioli, 1833), captured at west of San Antonio, $870 \mathrm{~mm}$ of LT, studied in March 1984 (non conserved). Under their mouth a spherical type protuberance was observed.

\section{AGRADECIMIENTOS}

El autor agradece al Sr. Raúl Leiva la donación de los ejemplares de B. polylepis de San Antonio y Cartagena ; a la Sra. Corina Silva y Sr. Henry Camera por el ejemplar de S. lobatus de Matanzas; al Sr. Juan Zúñiga por el ejemplar de M. mola; al Sr. Patricio Hernández y a los pescadores de San Antonio y Pesquera San Bernardo por los ejemplares de $M$. ramsayi; al Prof. Ismael Kong de la Universidad de Antofagasta, por la entrega de bibliografía, constante apoyo y estímulo, y revisión crítica del manuscrito; al Dr. Germán Pequeño de la Universidad Austral, por la revisión del manuscrito; al Prof. Nibaldo Bahamonde por impulsarme a publicar estos antecedentes y al personal del Museo de San Antonio por su valiosa colaboración.

\section{REFERENCIAS}

Atria, G. 1967. Un ectoparásito del pez luna (Mola ramsayi, Giglioli) Pennella cf. filosa L. (Crustacea, Copepoda). Not. Mens. Mus. Nac. Hist. Nat., Chile, 131: 3-5.

Bahamonde, N. 1963. Pez luna (Mola ramsayi) en Chiloé. Not. Mens. Mus. Nac. Hist. Nat., Chile, 82: 4-8.

Bahamonde, N. \& G. Pequeño. 1975. Peces de Chile. Lista sistemática. Mus. Nac. Hist. Nat., Chile, Publ. Ocas., 21: 1-20.

Berry, F. \& W. Baldwin. 1966. Triggerfishes (Balistidae) of the Eastern Pacific. Proc. Calif. Acad. Sci., 34(9): 429-474.

Bond, C.E. 1979. Biology of fishes. W.B. Saunders Co., Philadelphia, 514 pp.

Bond, C.E. 1997. Biology of fishes. Saunders Publishing Co., Fort Worth, 750 pp.

Chirichigno, N. 1974. Clave para identificar los peces marinos del Perú. Instituto del Mar del Perú, Callao, 44: 1-387.

Chirichigno, N. \& J. Vélez. 1998. Clave para identificar los peces marinos del Perú (Segunda edición, revisada y actualizada). Instituto del Mar del Perú. Publicación Especial, Callao, 500 pp.

De Buen, F. 1957. Los peces de la familia Molidae. Invest. Zool. Chil., 4: 66-76.

De Buen, F. 1959. Lampreas, tiburones, rayas y peces en la Estación de Biología Marina de Montemar. Rev. Biol.. Mar., Valparaíso, 9(1-3): 3-200.

Delfín, F.T. 1899. Catálogo de los peces de Chile. Rev. Chil. Hist. Nat., 4(3): 29-31.

Fischer, W. \& J.C. Hureau (eds.). 1985. FAO species identification sheets for fishery purposes. Southern Ocean (Fishing areas 48, 58 y 88) (CCAMLR Convention Area). Commission for the Conservation of the Antarctic Marine Living Resources, Rome, 2, $471 \mathrm{pp}$.

Fowler, H. 1945. Fishes of Chile. Systematic catalog. Rev. Chil. Hist. Nat., Parts I and II, pp. 36-171.

Fowler, H. 1951. Analysis of the fishes of Chile. Rev. Chil. Hist. Nat., 51-53: 263-326. 
Gotschlich, B. 1913. Llanquihue y Valdivia. Bol. Mus. Nac. Hist. Nat. Chile, 4: 78-240.

Hildebrand, S.F. 1946. A descriptive catalog of the shore fishes of Perú. Bull. U.S. Nat. Mus., 189: 195.

Kong, I., J. Tomicic \& J. Zegers. 1985. Ictiofauna asociada al fenómeno El Niño 1982-83 en la zona norte de Chile. Invest. Pesq., Chile, 32: 215-223.

Kong, I. \& A. Bolados. 1987. Sinopsis de peces asociados al fenómeno "El Niño" 1982-83 en el norte de Chile. Estud. Oceanol., 6: 25-58.

Mann, G. 1954. Vida de los peces en aguas chilenas. Instituto de Investigaciones Veterinarias y Universidad de Chile, Santiago, 342 pp.

Matsuura, K. 1980. A revision of Japanese Balistoid fishes. I. Family Balistidae. Bull. Nat. Sci. Mus., Ser. A (Zool.), 6(1): 27-69.

Meek, S. \& S. Hildebrand. 1928. The marine fishes of Panama. Publ. Field. Mus. Nat. Hist. Zool., Ser. 15(3): 709-1045.

Meléndez, R., O. Gálvez \& A. Cornejo. 1993. Catálogo colección de peces depositada en el Museo Nacional de Historia Natural, Chile. Mus. Nac. Hist. Nat., Chile, Publ. Ocas., 47: 1-224.

Moyle, P.B. \& J.C. Cech Jr. 1982. Fishes, an introduction to ichthyology. Prentice-Hall, New Jersey, 593 pp.

Oliver, C. 1930. Algunas observaciones sobre el pez luna (Mola mola) (Linn, Gilbert). Rev. Chil. Hist. Nat., 34: 200-207.

Oliver, C. 1943. Catálogo de los peces marinos del litoral de Concepción y Arauco. Bol. Soc. Biol., Concepción, 17: 75-126.

Recibido: 16 agosto 2002; Aceptado: 14 marzo 2003
Pequeño, G. 1984. Peces comunes marinos de Valdivia. Guía de reconocimiento para profesionales y aficionados del litoral. Universidad Austral de Chile, Valdivia, $62 \mathrm{pp}$.

Pequeño, G. 1989. Peces de Chile. Lista sistemática revisada y comentada. Rev. Biol. Mar., Valparaíso, 24(2): 1-132.

Pequeño, G. 1997. Peces de Chile. Lista sistemática revisada y comentada: addendum. Rev. Biol. Mar. Oceanogr., 32(2): 77-94.

Philippi, R.A. 1892. Algunos peces de Chile. Las rayas, Callorhynchus y Orthagoriscus chilenos. An. Mus. Nac. Chile. Zool., 9: 1-16.

Porter, C. 1904. Sobre el Orthagoriscus mola. Rev. Chil. Hist. Nat., 8: 238-239.

Quijada, B. 1913. Catálogo ilustrado y descriptivo de la colección de peces chilenos y extranjeros. Bol. Mus. Nac. Hist. Nat., 13: 280-293.

Reed, E. 1897. Catálogo de los peces chilenos. An. Univ. de Chile, 98: 653-673.

Springer, V.G. 1982. Pacific plate biogeography, with special reference to shore fishes. Smith. Contr. Zool., 367: 4-182.

Vélez, J., J. Cevallos \& M. Méndez. 1984. Effects of 1982-83 El Niño on fishes and crustaceans off Perú. Trop. Ocean-Atmosph. News., 28: 10-12.

Villalba, C. \& J. Fernández. 1985. Parásitos de Mola ramsayi (Giglioli, 1883) (Pisces: Molidae) en Chile. Bol. Soc. Biol., Concepción, 56: 71-78. 